\title{
CARBOIDRATOS COMO FONTE DE COMPOSTOS PARA A INDÚSTRIA DE QUÍMICA FINA
}

\author{
Vitor Francisco Ferreira* e Fernando de Carvalho da Silva \\ Departamento de Química Orgânica, Universidade Federal Fluminense, Campus do Valonguinho, 24020-141 Niterói - RJ, Brasil \\ Patricia Garcia Ferreira \\ Faculdade de Farmácia, Universidade Federal Fluminense, 24241-000 Niterói - RJ, Brasil
}

Recebido em 4/7/13; aceito em 10/10/13; publicado na web em 24/10/13

\begin{abstract}
CARBOHYDRATES AS A SOURCE OF COMPOUNDS FOR FINE CHEMICAL INDUSTRY. Coal, oil, natural gas, and shale gas are biomass that is formed millions of years ago. These are non-renewable and depleting, even considering the recent discovery of new sources of oil in the presalt and new technologies for the exploitation of shale deposits. Currently, these raw materials are used as a source of energy production and are also important for the production of fine chemicals. Since these materials are finite and their (oil) price is increasing, it is clear that there will be a progressive increase in the chemical industry to use renewable raw materials as a source of energy, an inevitable necessity for humanity. The major challenge for the society in the twenty first century is to unite governments, universities, research centers, and corporations to jointly act in all areas of science with one goal of finding a solution to global problems, such as conversion of biomass into compounds for the fine chemical industry.Non-renewable raw materials are used in the preparation of fuels, chemical intermediates, and derivatives for the fine chemical industry. However, their stock in nature has a finite duration, and their price is high and will likely increase with their depletion. In this scenario, the alternative is to use renewable biomass as a replacement for petrochemicals in the production of fine chemicals. As the production of biomass-based carbohydrates is the most abundant in nature, it is judicious to develop technologies for the generation of chain products (fuels, chemical intermediates, and derivatives for the fine chemicals industry) using this raw material. This paper presents some aspects and opportunities in the area of carbohydrate chemistry toward the generation of compounds for the fine chemical industry.
\end{abstract}

Keywords: fine chemicals; chains fine chemical from carbohydrates; renewable biomasses.

\section{INTRODUÇÃO}

A compreensão humana sobre a natureza e a vida é baseada em nosso conhecimento da química, pois, em realidade, todos os processos da vida são controlados por reações químicas.

Há vários problemas globais ${ }^{1,2}$ em que a química pode ser o elemento chave de sua solução e, desta forma, promover o avanço social e econômico dos países em um mundo sem fronteiras. A saber:

1. biodiversidade e conversão de biomassa em matérias-primas;

2. fontes alternativas de energia limpa e renovável;

3. produção sustentável de fármacos;

4. a produtividade agrícola para alimentar o crescimento populacional;

5. qualidade da água para uso humano;

6. materiais reclicáveis e pouco persistentes;

7. nível dióxido de carbono e seu aproveitamento;

8. risco de desaparecimento de alguns elementos químicos por dispersão;

9. nanotecnologia e nanotoxicologia;

10. educação em todos níveis e em especial em química e;

11. responsabilidade social da química.

O grande desafio da sociedade no século XXI é unir governos, universidades, centros de pesquisa e corporações para que atuem conjuntamente em todas as áreas da ciência com uma única meta encontrar soluções para os problemas globais, como por exemplo, conversão das biomassas em compostos para a indústria de química fina.

\footnotetext{
*e-mail: cegvito@vm.uff.br
}

\section{DISCUSSÃO}

O carvão, petróleo, gás natural e o gás de xisto são biomassas formadas há milhões de anos atrás, não-renováveis e tende ao desaparecimento, mesmo com as recentes descobertas de novas fontes de petróleo na região do pré-sal e as novas tecnologias para o aproveitamento das jazidas de xistos. Atualmente essas matérias-primas são utilizadas como fonte de produção de energia ${ }^{3-5}$ e também são importantes insumos para a fabricação de produtos da química fina. ${ }^{6,7}$ Esses materiais fósseis são finitos e seu preço está em um patamar elevado, logo, fica evidente que haverá um aumento progressivo da indústria química com base em matérias-primas renováveis como uma necessidade inevitável para a humanidade. ${ }^{8}$ Conjugado a essas previsões está o fato real de que a demanda de energia nos próximos 50 anos atingirá cerca de 30 a 60 TW.

Dentro deste cenário, as biomassas renováveis surgem como insumos que tomaram o lugar destes materiais não-renováveis, pois são formadas por reações fotossintéticas, ou seja, são materiais que fixam a energia solar em compostos químicos, normalmente em biopolímeros de alta massa molecular (ex: amido, quitina e celulose). Em realidade, as biomassas são materiais orgânicos disponíveis em uma base renovável, incluindo árvores, resíduos agroindustriais (bagaço de cana, serragem, resíduos de papel, grãos utilizados nas cervejarias, palhas, caules, frutos, talos, folhas, etc.), madeira, resíduos de processamento (glicerol) e resíduos alimentares (bagaço de laranja, pó de café, casca de arroz, casca de coco), resíduos de proteínas, plantas aquáticas, dejetos animais (esterco), esgoto terciário e outros materiais residuais das indústrias. Enquanto o petróleo não está distribuído no mundo de forma de que todos os países possam aproveitar seu potencial, as biomassas estão presentes em todos os países de forma diversificada. Países com grandes quantidades de recursos naturais tem o potencial para se tornarem os líderes mundial em termos de 
economia sustentável e as suas tecnologias (ou soluções) poderão servir ao mundo (Figura 1).

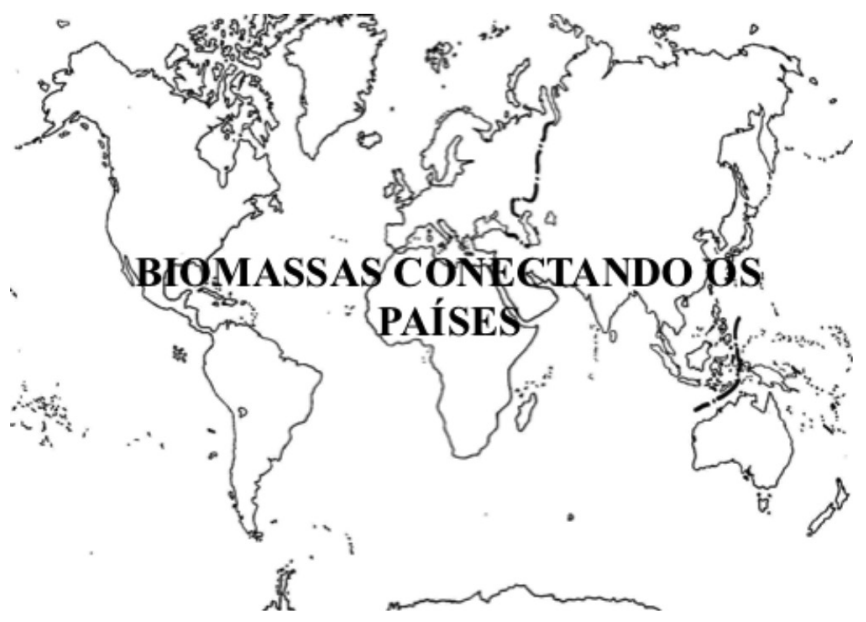

Figura 1. As biomassas como materiais conectando os países

Dentre as biomassas, destaca-se a classe dos carboidratos que são as biomoléculas formadas primeiramente no processo de fotossíntese, mas que por biotransformações intracelulares eles são transformados em outras biomassas (Figura 2).

A chamada biomassa renovável terrestre é constituída de diversos produtos de baixas e altas massas moleculares e de diversas naturezas químicas, como por exemplo, açúcares, aminoácidos, terpenos, lipídios, e biopolímeros de altas massas moleculares, como celulose, hemicelulose, quitina, amido, lignina e proteínas. A posição do Brasil é extremamente vantajosa, pois ele tem extensão continental privilegiada e de excelente qualidade em termos de água e solo. Portanto, tem-se um elevado potencial de produção de matérias-primas energéticas, como o álcool, o biodiesel e a biomassa sólida, que são também importantes insumos da indústria química. ${ }^{9}$

\section{Biomassa Renovável}

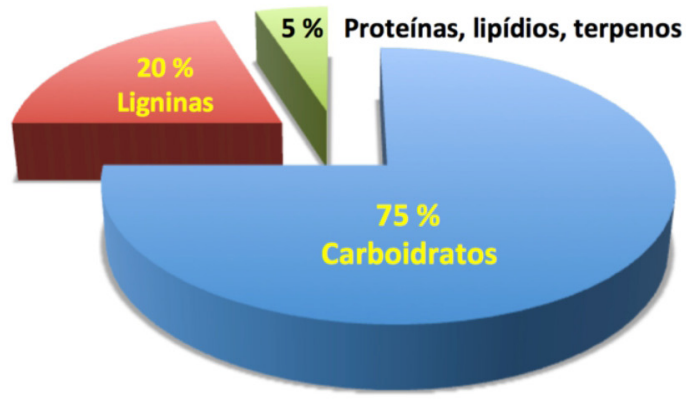

Figura 2. Distribuição das biomassas renováveis

De uma forma geral os produtos naturais formam cadeias produtivas importantes para a economia dos países, ou mais modernamente são importantes para a economia verde. No caso das biomassas que são em produzidas larga escala pela natureza podem ter três as possíveis destinações: alimentação, ${ }^{10,11}$ produção de energia (biocombustíveis) e fabricação de produtos para a química fina.

A energia produzida por fontes renováveis representa apenas $8 \%$ da energia total produzida no mundo e dentro desse conjunto a energia produzida por biomassas representa 38\%. Tendo em vista que as fontes hidroelétricas (55\%) tendem a uma estabilização por diversas questões, isso indica que as biomassas como fonte renovável de energia tendem a crescer (Figura 3). ${ }^{12}$

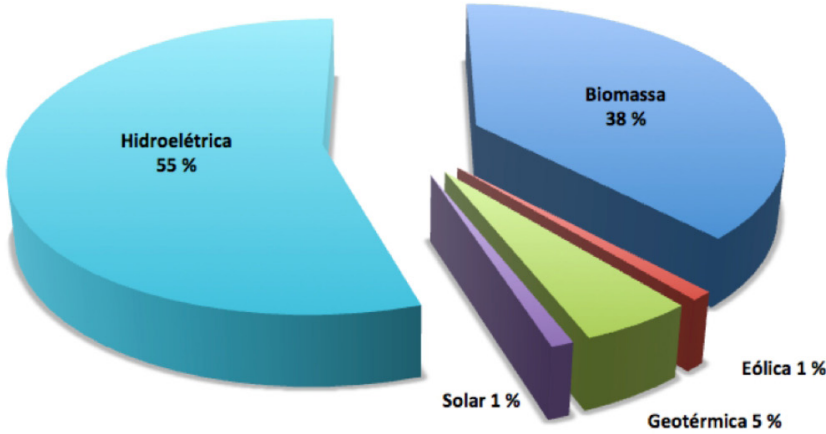

Figura 3. Distribuição percentual das fontes de energia renováveis

Apenas para contextualizar, os produtos da química fina são matérias-primas de alta qualidade e especialização, cuja produção anual fica abaixo das 10 mil toneladas e preço acima de US\$ 5.000. Incluemse nessa classe, por exemplo, fragrâncias, agroquímicos, produtos farmacêuticos, dentre muitos outros de aplicações industriais. Eles diferem dos chamados intermediários químicos que são produzidos em grandes quantidades utilizando procedimentos padronizados e com produção anual acima de 10 mil toneladas. No entanto, seus preços são mais baixos, ficando entre US\$ 1.500-2.500/ton. Como exemplo tem-se os produtos oriundos da petroquímica, como etileno, propileno, aromáticos, etc.

Os carboidratos compõem $75 \%$ da biomassa da Terra representando a maior fonte renovável do planeta e anualmente são produzidos cerca de 200 mil toneladas em carboidratos pelas plantas superiores e algas. ${ }^{13-15}$ Porém, é importante destacar que dentro dessa classe estão incluídas muitas substâncias, mas poucas são as que efetivamente tem o potencial econômico para atrair o investimento das indústrias químicas. ${ }^{16}$ Considera-se que em realidade são quatro carboidratos que têm esse potencial econômico desejado para produção de compostos químicos: celulose, amido, quitina e sacarose (Figura 4).
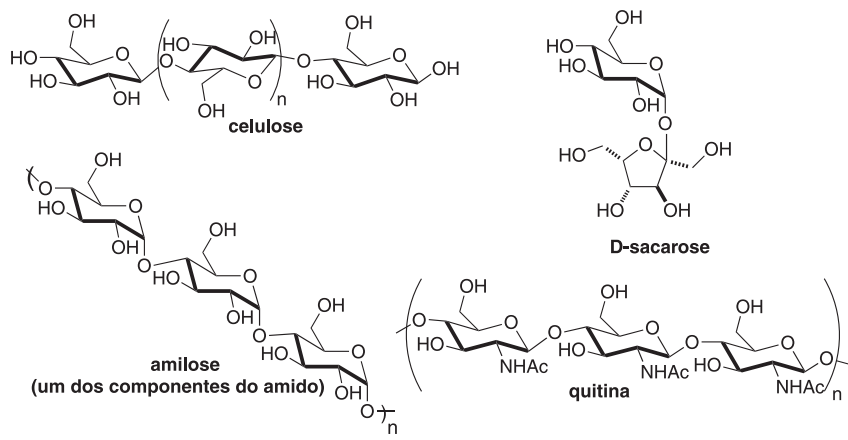

Figura 4. Carboidratos com potencial econômico para as indústrias químicas

Dos carboidratos mostrados na Figura 4, a celulose e amido são hidrolisados à D-glicose em grandes quantidades no mercado. O mesmo é observado para a quitina, que produz a D-glicosamina. ${ }^{17}$ Portanto, esses carboidratos, associados a D-frutose e o D-manitol, são monossacarídeos que também são atrativos como matéria prima abundante e de preço bastante acessível para as indústrias químicas (Figura 5). ${ }^{18}$ Já existem processos químicos e biotecnológicos que podem transformar esses carboidratos em compostos orgânicos
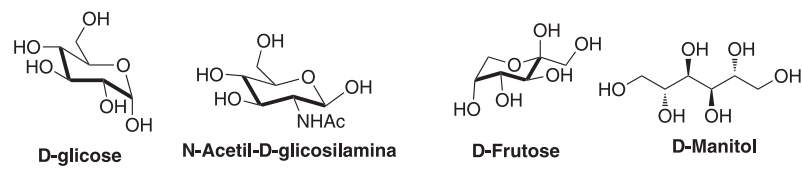

Figura 5. Importantes monossacarídeos produzidos em escala industrial 
iguais aos compostos oriundos do petróleo, em quantidades capazes de atrair as indústrias para a produção de compostos de interesse da indústria de química fina. ${ }^{19,20}$

Dentro dessa linha de discussão sobre usos de carboidratos como fontes renováveis para novos compostos importantes não se pode deixar de comentar sobre os relatórios produzidos pelo "Pacific Northwest National Laboratory" do "US Department of Energy (DOE)" ${ }^{21,22}$ onde, no relatório de 2004, são recomendados quinze blocos de construção produzidos a partir de diversos carboidratos por conversões biológicas ou químicas abundantes que podem ser transformados em produtos químicos de alto valor agregado ou materiais de base biológica. A seleção desses blocos levou em consideração moléculas com múltiplos grupos funcionais, possibilidade de produção em larga escala, desafios e complexidade destas vias e que poderiam ser transformados em novas famílias secundárias de moléculas importantes para a indústria. Os blocos de construção propostos são: ácido fumárico, succínico, málico, 2,5-furano dicarboxílico, 3-hidroxi-propiônico, aspártico, glucárico, glutâmico, itacônico e levulínico, o 3-hidroxibutirolactona glicerol, o sorbitol e o xilitol/arabinitol. Com a tecnologia atual, em nossa opinião, pelo menos mais alguns outros compostos poderiam ser incluídos nessa lista como os ácidos cítrico e glucônico e, também, o hidroximetil furfural a levoglucosana (Figura 6). Nada impede que a lista continue crescendo com o advento de novos processos por a serem desenvolvidos por biologia sintética. ${ }^{23}$

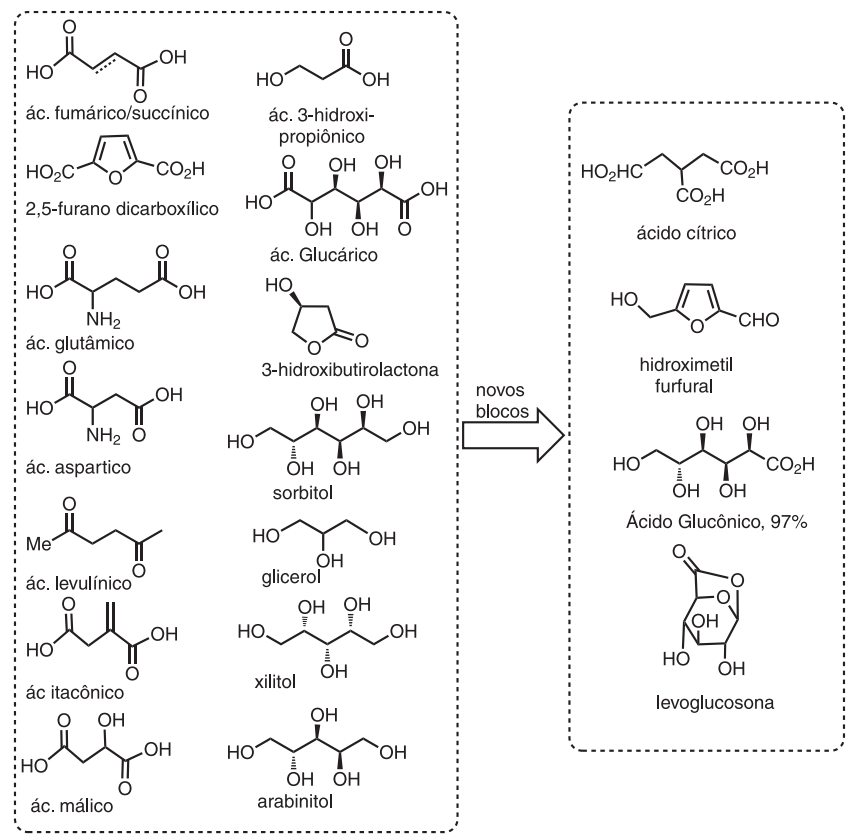

Figura 6. Quinze blocos de construção produzidos a partir de diversos de carboidratos

A sacarose é a D-glicose são os dois carboidratos de baixa massa molecular, economicamente atrativos e produzidos em larga escala e que são propensos a modificações estruturais para a produção de novos compostos importantes do ponto de vista industrial (Figura 7).

A sacarose é o carboidrato de baixa massa molecular produzido em larga escala por diversos países, principalmente da cana-de-açúcar, que tem um dos mais eficientes mecanismos fotossintéticos fixação da radiação solar. A produção global de açúcar estimada para 2013/14 é de 175 milhões toneladas cúbicas ${ }^{24}$ com tendências ao crescimento até $2021 .{ }^{25}$ O Brasil e a Tailândia são os maiores produtores e os preços internacionais estão em níveis mais baixos que há três anos. Entretanto, a diferença entre a produção global de açúcar e seu consumo está se estreitando devido aos usos alimentares e a produção de etanol. A D-glicose, apesar de ser uma das unidades da sacarose, tem o amido como sua principal fonte de produção. Cerca de $34 \%$ do amido produzido no mundo ${ }^{26,27}$ é transformado em glicose.

A partir de processos químicos ou biocatalíticos em escala industrial ${ }^{28,29}$ é possível transformar a sacarose e D-glicose em muitos outros carboidratos, ${ }^{30-33}$ produtos para diversos fins de aplicações diretas (hidrogéis, ${ }^{34-36}$ dextranas, ${ }^{37}$ extração de petróleo, ${ }^{38}$ adoçantes artificiais, ${ }^{39-42}$ tensoativos ${ }^{43}$ resinas, ${ }^{44,45}$ polímeros, ${ }^{46-48}$ etc.), solventes e compostos químicos úteis para a indústria de química fina (Figura 6) e, portanto, compostos com maior valor agregado e grande interesse econômico. ${ }^{49,50}$ Cabe ressaltar que o composto obtido em maior quantidade a partir da sacarose é o etanol, que não se enquadra dentro dessa classificação de compostos químicos úteis para a indústria de Química, a não ser como solvente verde para reações, mas ele per se constitui uma cadeia de outros compostos químicos e intermediários para polímeros. ${ }^{51}$ Como se pode denotar do pequeno resumo esquemático mostrado na Figura 7, diversos blocos de construção propostos podem ser obtidos a partir da sacarose, como os ácidos succínico, málico, fumárico, 2,5-furano dicarboxílico, 3-hidroxi- propiônico, aspártico, glucárico, glutâmico, itacônico, levulínico e ainda o 3-hidroxibutirolactona glicerol, o sorbitol e o xilitol/arabinitol, além de outros ácidos carboxílicos, incluindo aminoácidos importantes tanto para a alimentação como para outras transformações de interesse das indústrias de química fina.

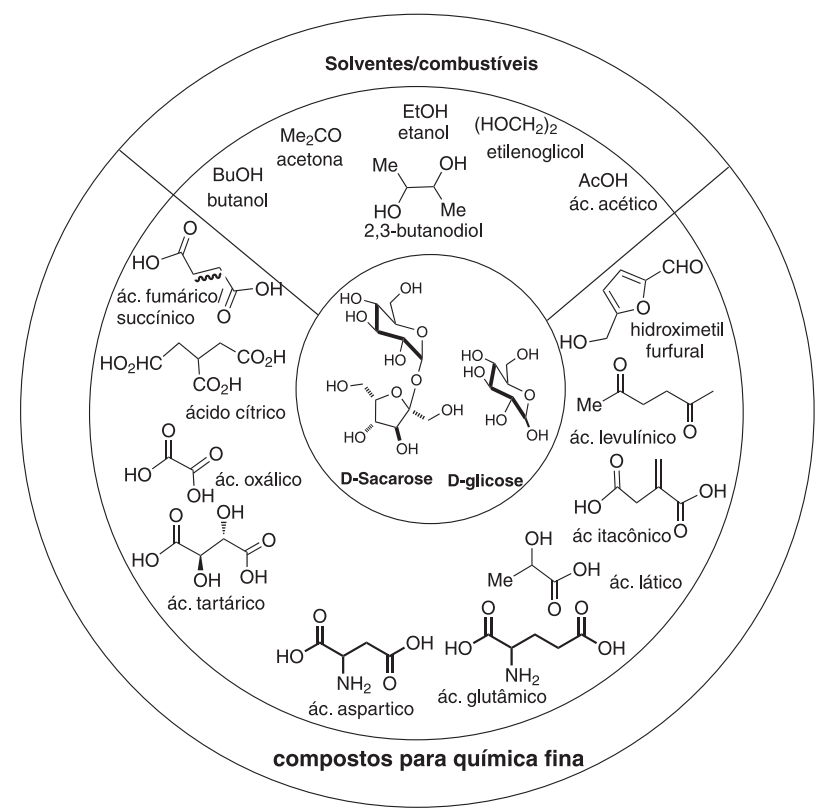

Figura 7. Alguns compostos possíveis de serem obtidos pelas rotas da D-sacarose elou D-glicose

A D-glicose é uma das maiores cadeias de transformação de um carboidrato em outros carboidratos e, consequentemente, em outras cadeias secundárias. Existe uma variedade de insumos industriais como, por exemplo, sorbitol, manose, sorbose, frutose, dextrinas, dentre outros, obtidos em escala industrial por processos químicos ou fermentativos (aeróbicos e anaeróbicos), ${ }^{52,53}$ que são úteis para obtenção de alcoóis, polialcoóis, aminoácidos, ácidos carboxílicos (como ácido cítrico, lático e acético) e até mesmo a vitamina C. Estudos recentes mostram o desempenho de cianobactérias na digestão de biomassas enriquecidas de carboidratos na produção de biometano ${ }^{54}$ e bioetanol por microalgas. ${ }^{55} \mathrm{~A}$ importância desse carboidrato advém 
da sua versatilidade em ser transformadas em substâncias acíclicas, furanosídicas ou piranosídicas, ${ }^{56}$ além de derivados facilmente obtidos utilizando-se reações simples tais como oxidação, redução, aminação, cianidação, eterificação, glicosidação, esterificação, isomerização, epimerização e formação de acetonídeos.$^{57,58} \mathrm{~A} \delta$-gluconolactona, que pode ser obtida a partir ácido D-glucônico com $97 \%$ de rendimento por oxidação do carbono anomérico, ${ }^{59-62}$ tem um produção mundial de cerca de 30.000.000 ton/ano. Seus sais são fármacos ou transformados em química fina como a $\delta$-lactona, que per se é um novo nó na rede da D-glicose, mas que tem produção e usos diversificados no mercado (Figura 8).

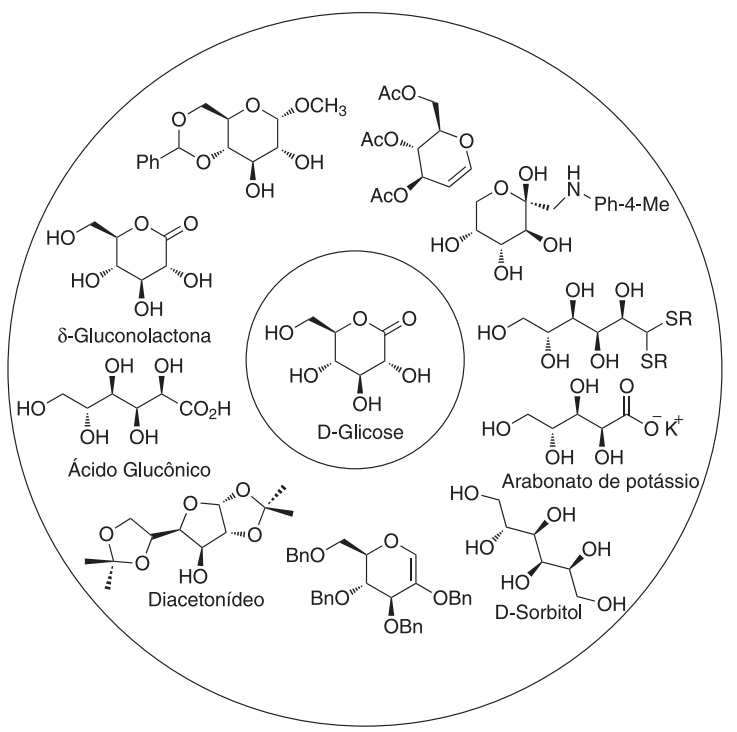

Figura 8. Alguns carboidratos obtidos a partir da D-glicose de interesse industrial

Não é a intenção desse trabalho realizar uma completa varredura de todos os compostos considerados blocos de construção para possíveis composto para a química fina. Por escolha dos autores, decidiu-se por alguns que estão na lista entre os que são considerados mais promissores, mas também por outros que começam aparecer na literatura como importantes, a saber: ácidos aspártico, glutâmico, levulínico e 5-hidroximetilfurfural (HMF).

O ácidos L-aspártico e L-glutâmico podem ser obtidos a partir da D-sacarose ou D-glicose por meio da síntese química, da extração de proteínas, da fermentação, e da conversão enzimática (Figura 9). Esses aminoácidos diferem entre si por apenas um grupo $\mathrm{CH}_{2}$. No entanto, o método de obtenção preferido de ácido L-aspártico é a aminação enzimática, com células de Escherichia coli imobilizadas, do ácido fumárico, pois forma menos subprodutos e apresenta uma grande facilidade de separação por cristalização. ${ }^{63-65}$

Por outro lado, o método de obtenção de monossódio L-glutamato, um atrativo potencializador do sabor, é a partir de um processo fermentativo com a bactéria do solo Corynebacterium glutamicum, que é capaz de produzir ácido L-glutâmico em altos rendimentos a partir da sacarose ou glicose. Por meio desse processo o ácido L-glutâmico é fabricado em 2,6 milhão de toneladas por ano, sendo considerado o aminoácido com maior capacidade de produção no mundo. ${ }^{66-69}$

O principal aspecto que tornou esses aminoácidos importantes para a química fina foi a capacidade de se fazer reduções seletivas na presença de outras funcionalidades, mais especificamente a conversão dos grupos ácidos na presença de aminas por processos catalíticos. No entanto, ainda há espaço para estudos mais aprofundados dos processos de hidrogenação/redução química para a conversão dos ácidos aspártico e glutâmico para os análogos mostrados na Figura 9. Para isso é importante se desenvolver novos sistemas catalíticos que envolvam também a função amina.

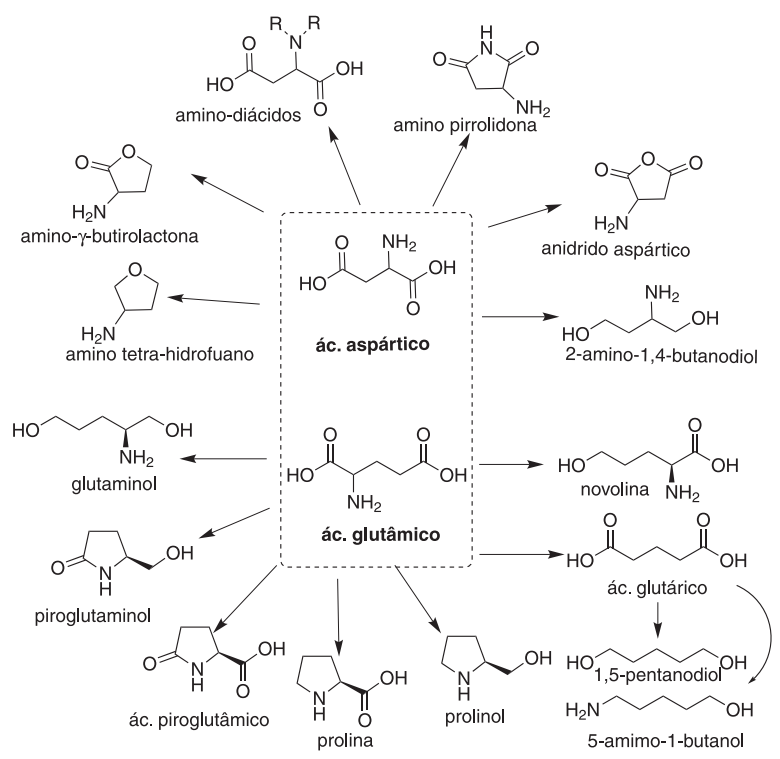

Figura 9. Cadeia de produtos obtidos a partir dos ácidos L-aspártico e L-glutâmico

O ácido levulínico é extremamente vantajoso, pois é um potencial gerador de produtos da química fina por ter três funcionalidades bem distintas. Ele pode ser preparado por diversas rotas, sendo uma delas a partir da D-sacarose por aquecimento em meio de um ácido forte concentrado. ${ }^{70} \mathrm{O}$ processo envolve a D-glicose que isomeriza à D-frutose e, por desidratação, leva ao hidroximetilfurfural (HMF). Obviamente o ácido levulínico pode ser preparado a partir da própria D-frutose ou do $\mathrm{HMF}^{71}$ mas outros carboidratos também podem ser utilizados nesta degradação ácida como a inulina e amido. ${ }^{72,73}$ Essa é uma das principais aplicações da D-frutose fora do contexto alimentar.

Os compostos contendo o fragmento furânico em sua estrutura, tais como o 5-hidroximetilfurfural (HMF) e furfural, são importantes para a produção de composto da química fina. Eles podem ser obtidos de muitas biomassas renováveis baseadas em carboidratos como lignoceluloses, por exemplo. ${ }^{74}$ Apesar dessas duas substâncias não estarem entre os blocos sintéticos de construção de moléculas e produtos substitutos do petróleo ${ }^{75}$ eles apresentam grande utilidade. Esses derivados são intermediários potencialmente importantes do ponto de vista industrial e que podem ser produzidos em larga escala. Destes, o ácido 5-hidroximetilfuróico, o ácido 2,5-dicarboxílico (contido na plataforma de compostos de interesse para a química fina), a 1,6-diamina e o 1,6-diol são os intermediários mais versáteis e de elevado potencial industrial, uma vez que são monômeros de seis carbonos que podem substituir o ácido adípico, os alquildióis e a hexametillenodiamina na produção de poliamidas e poliésteres (Figura 10).

\section{CONCLUSÃO}

“A Química fina obtida a partir de carboidratos terá peso importante na balança comercial dos países que investirem nestas tecnologias"

As biomassas baseadas em carboidratos oferecem grandes oportunidades para o Brasil, que é potencialmente um gerador de diversos 


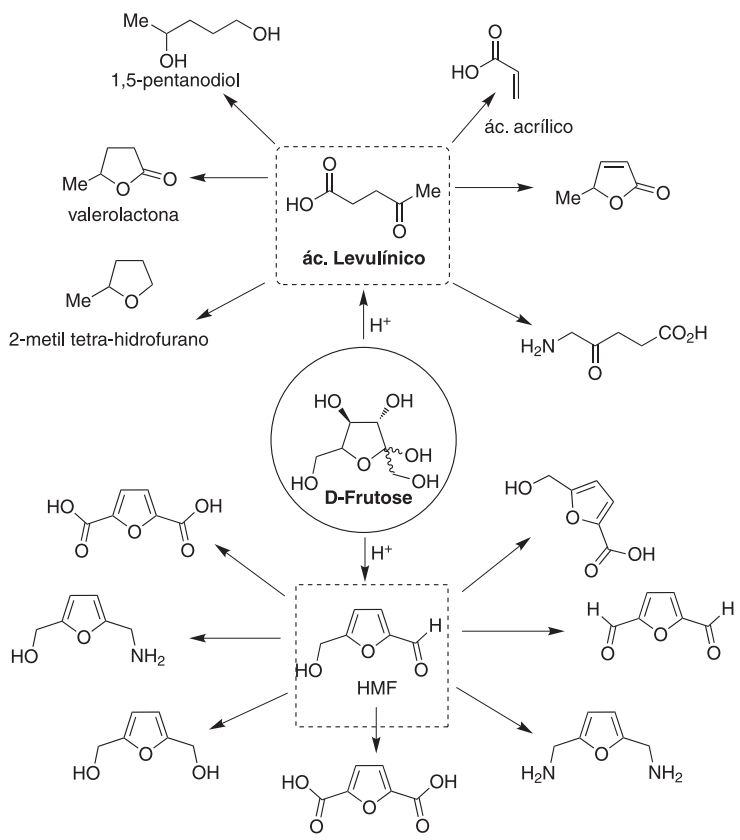

Figura 10. Ácido levulínico e HMF como precursores de derivados obtidos da D-Frutose

tipos de biomassas das quais se podem produzir insumos químicos básicos e intermediários da química fina. Neste trabalho foram mostradas as potencialidades de alguns carboidratos na produção de compostos para a química fina: ácidos aspártico, glutâmico, levulínico e 5-hidroximetilfurfural (HMF). As oportunidades estão abertas, pois é preciso desenvolver novas tecnologias a partir de biomassas para enfrentamento da era pós-petróleo, pois não há dúvidas de que a biomassa terrestre ocupará um dos vértices das opções de fonte alternativa de energia, continuará atuando fortemente na alimentação e deverá se a principal fonte de matéria-prima para a produção de produtos de interesse das indústrias de química fina. Considerando esses aspectos, os compostos apresentados nesse texto, obtidos a partir de carboidratos abundantes, produzirão outras substâncias importantes e que serão plataformas para outras cadeias de intermediários. É preciso que as empresas se conscientizem da importância das biomassas como recursos renováveis de nova tendência e invistam em novas tecnologias para seu uso.

\section{AGRADECIMENTOS}

Ao Prof. J. B. Andrade (UFBA) e à Sociedade Brasileira de Química pelo convite e ao Departamento de Química Orgânica da UFF por todo apoio.

\section{REFERÊNCIAS}

1. Ferreira, V. F.; Quím. Nova 2006, 29, 3.

2. Santos, A. P. B.; Pinto, A. C.; Química Nova na Escola 2008, 1.

3. Suarez, P. A. Z.; Meneghetti, S. M. P.; Ferreira, V. F.; Quim. Nova 2006, 29, 1157.

4. Pinto, A. C.; Guarieiro, L. L. N.; Rezende, M. J. C.; Ribeiro, N. M.; Torres, E. A.; Lopes, W. A.; Pereira, P. A. P.; Andrade, J. B.; J. Braz. Chem. Soc. 2005, 16, 1313.

5. Galembeck, F. Em Brazilian Network on Green Chemistry: Awareness, Responsibility and Action; Carioca, J. O. B., ed.; UFC: Fortaleza, 2008, p. 217.

6. Kamm, B.; Kamm, M.; Gruber, P. R.; Kromus, S. Em Biorefineries Industrial Processes and Products. Status Quo and Future Directions;
Kamm, B.; Gruber, P. R.; Kamm, M., eds.; Wiley-VCH Verlag GmbH \& Co. KGaA: Weinheim, 2006, vol. 1, 3.

7. Storcker, M.; Angew. Chem. Int. Ed. 2008, 47, 2.

8. Galembeck, F.; Alves, O. L.; J. Braz. Chem. Soc. 2010, 21, 947.

9. Pinheiro, S.; Ferreira, V. F.; Quim. Nova 1998, 21, 312.

10. Bols, M.; Carbohydrate building blocks, John Wiley \& Sons, Inc.: New York, 1996

11. http://www.eia.gov/totalenergy/data/annual/pdf/aer.pdf, acessada em 20/6/2013.

12. Ferreira, V. F; Quim. Nova 1995, 18, 267.

13. Lichtenthaler, F. W. Em Biorefineries, Industrial Processes and Products; Kamm, B.; Gruber, P. R.; Kamm, M., eds.; Wiley-VCH: Weinheim, 2006, Vol. 2, 3; Lichtenthaler, F. W.; Carbohydrates, 6th ed., Ullmann's Encyclopedia of Industrial Chemistry; 2002; Lichtenthaler, F. W. Em Carbohydrates as Raw Materials for the Chemical Industry, Tundo, P, ed. Green Chemistry Series, 3rd ed., INCA: Venezia, 2004, 105-127; Lichtenthaler, F. W.; Peters, S.; C. R. Chim. 2004, 7,65 .

14. Lichtenthaler, F. W.; Acc. Chem. Res. 2002, 35, 728.

15. Okkerse, C.; van Bekkum, H.; Green Chem. 1999, 1, 107.

16. Filho, S. P. C.; Signini, R.; Cardoso, M. C.; Revista Processos Químicos 2007, 2, 9 .

17. Stevens, C. V.; Verhé, S. R.; Renewable Bioresources Scope and Modification for Non-food Applications, Eds. C. V.; John Wiley \& Sons: Chichester, 2004.

18. Schuchardt, U.; Ribeiro, M. L.; Gonçalves, A. R.; Quim. Nova 2004, 24, 247.

19. Leite, A. L. S.; Laeal, M. R. L. V. Em Brazilian Network on Green Chemistry: Awareness, Responsibility and Action; Carioca, J. O. B., ed.; UFC: Fortaleza, 2008, 217.

20. Werpy, G. Petersen (eds.); Top Value Added Chemicals from biomass (ed) [U.S. Department of Energy, Office of scientific and technical information, 2004, No.: DOE/GO-102004-1992], http://www1.eere.energy. gov/biomass/pdfs/35523.pdf, acessada em Junho de 2013.

21. Holladay, J. E.; Bozell, J. J.; White, J. F.; Johnson, D.; Top Value-Added Chemicals from Biomass Volume II-Results of Screening for Potential Candidates from Biorefinery Lignin; http://www1.eere.energy.gov/ biomass/pdfs/pnnl-16983.pdf, acessada em Junho de 2013.

22. Chan S.; Kanchanatawee, S.; Jantama, K.; Bioresour. Technol. 2012, 103, 329 .

23. United States Department of Agriculture; Sugar: World Markets and Trade, http://www.fas.usda.gov/psdonline/circulars/sugar.pdf, acessada em Maio 2013.

24. Koo, W. W.; Taylor, R. D.; 2012 Outlook of the U.S. and World Sugar Markets, 2011-2021, http://ageconsearch.umn.edu/bitstream/128037/2/ AAE692.pdf, acessada em Junho de 2013.

25. Roper, von H.; Koch, H.; Starch-Starke 1988, 40, 121.

26. Roper, von H.; Koch, H.; Beck, R.; Starch-Starke 1993, 45, 121

27. Willians, R.; Sucrochemistry: Chemical Fermentation; R. F. Gould, ed.; ACS Symposium Series 41, 1977, 274.

28. James, C. E.; Hough, L.; Khan, R.; Prog. Chem. Org. Nat. Prod. 1989, $55,117$.

29. Daud, D.; Remaud-Sim, M.; Andre, I.; Nat. Prod. Rep. 2012, 29 , 945.

30. Lichtenthaler, F. W.; Eur. J. Org. Chem. 2002, 4095.

31. Lichtenthaler, F. W. Em Methods and Reagents for Green Chemistry: An Introduction; Tundo, P.; Perosa, A.; Zecchini, F., eds.; John Wiley \& Sons. Inc.: New Jersey, 2007, 23.

32. Ferreira, V. F; da Rocha, D. R.; da Silva, F. C.; Quim. Nova 2009, 32, 623.

33. Patil, N. S.; Dordick, J. S.; Rethwisch, D. G.; Biomaterials 1996, 17, 2343; Chen, J.; Park, K.; Carbohyd. Polym. 2000, 41, 259; Shantha, K. L.; Harding, D. R. K.; J. Appl. Polymer Sci. 2002, 84, 2597. 
34. Zhang, J.; Wang, A.; React. Funct. Polym. 2007, 67, 737.

35. Hoffman, A. S.; Adv. Drug Deliv. Rev. 2002, 43, 3; Pourjavadi, A.; Harzandi, A. M.; Hosseinzandeh, H.; Eur. Polym. J. 2004, 40, 1363; Mooney, D.; Lee, K. Y.; Chem Rev. 2001, 101, 1869.

36. Dextran é um grupo de polissacarídeos de alta massa molecular composto de cadeias de unidades D-glicose que são sintetizados a partir de D-sacarose.

37. Roller, S.; Dea, I. C. M.; Crit. Rev. Biotechnol. 1992, 12, 261; Qader, S. A. U.; Iqbal, L.; Aman, A.; Shireen, E.; Azhar, A.; Turk. J. Biochem. 2005, 31, 21; Sarwat, F.; Qader, S. A. U.; Aman, A.; Ahmed, N.; Int. J. Biol. Sci. 2008, 4, 379.

38. Hough, L.; Int. Sugar J. 1989, 91, 23.

39. Khan, R. Em Sucrose. Properties and applications; Mathlouthi, M.; Reiser, P., eds.; Blackie Academic \& Professional: Glasgow, 1995, cap. 11.

40. Mann, S. W.; Yuschak, M. M.; Amyes, S. J. G.; Aughton, P.; Finn, J. P.; Food Chem. Toxicol. 2000, 38, S91.

41. Hough, L.; Phadnis, S. P.; Khan, R.; Jenner, M. R.; Brit. Pat. 1.543.167, 1977. (CA 87: 202019v).

42. Mackenzie, K. M.; Tisdel, P. J.; Hall, R. L.; Boysen, B. G.; Field, W. E.; Chappel, C. I.; Food Chem. Toxicol. 1998, 36, 111.

43. Sachinala, N. D.; Litt, M. H.; US Patent 5646226, 1997.

44. Sachinvala, N. D.; Winsor, D. L.; Menescal, R. K.; Ganjian, I.; Niemczura, W. P.; Litt, M. H.; J. Polym. Sci., Part A: Polym. Chem. 1998, 36, 2397.

45. Frisch, K. C.; Kresta, J. E., Sucrochemistry: An Overview of Sugar Uretanes, Gould, R. F., ed; ACS Symposium Series 41, 238, 1977.

46. Chheda, J. N.; Roman-Leshkov, Y.; Dumesic, J. A.; Green Chem. 2007, 9,342 .

47. Chheda, J. N.; Dumesic, J. A.; Catal. Today 2007, 123, 59.

48. Kollonitsch, V.; Sucrose Chemicals, International Sugar Research Foundation: Washington D.C., 1970.

49. Buchholz, K. E., Stoppok, E., Matalla, K., Reh, K. D.; Jördening, H.-J.; Carbohydrates as Organic Raw Materials, Lichtenthaler, F. W. ed.; VCH Verlag: New York, 1991, p. 155.

50. Carioca, J. O. B. (ed.), Brazilian Network on Green Chemistry: Awareness, Responsibility and Action, UFC: Fortaleza, 2008.

51. Röper, H.; Starch-Stärke 2002, 54, 89.

52. Lichtenthaler, F. W.; Peters, S.; C. R. Chim. 2004, 7, 65.

53. Markou, G.; Angelidaki, I.; Georgakakis, D.; Fuel 2013, 111, 872.
54. Ho, S.-H.; Huang, S.-W.; Chen, C.-Y.; Hasunuma, T.; Kondo, A.; Chang, J.-S.; Bioresour. Technol. 2013, 135, 191.

55. Hanessian, S.; Total Synthesis of Natural Products. The Chiron Approach, Pergamon Press: Oxford, 1983.

56. Stevens, C. V. Em Renewable Bioresources - Scope and Modification for Non-food Applications; Stevens, C. V.; Verhé, R., eds.; John Wiley \& Sons Inc.: New Jersey, 2004, p. 160.

57. Kunz, H.; Ruck, K.; Angew. Chem. Int. Ed. 1993, 32, 336.

58. Reis, M. I. P.; Mendes, M. T.; da Silva, F. C.; Ferreira, V. F.; Revista Virtual de Química 2011, 3, 247.

59. Ferreira, V. F.; Pérez, L. M.; Nakamura, T.; Nakamura, L. K.; Pat. Brasil 9201473, 1992

60. Wit, G. D.; Vlieger, J. J. D.; Kockvandalen, A. C.; Kieboom, A. P. G.; Vanbekkum, H.; Tetrahedron Lett. 1978, 15, 1327.

61. Hepner, L.; Carbohydrate feedstock for the fermentation industry, Associates Ltda: London, 1988.

62. Szymanska, G.; Sobierajski, B.; Chmiel, A.; Polish J. Microbiol. 2011, $60,105$.

63. Huang, J.; Jin, N.; Katsuda, T.; Fukuda, H.; Yamaji, H.; Biochem. Eng. J. 2009, 46, 65 .

64. Aydogan, O.; Bayraktar, E.; Parlaktuna, M.; Mehmetoglu, T.; Mehmetoglu, U.; Biocatal. Biotransform. 2007, 25, 365.

65. Third quarter-FY2012 Market and other information, Ajinomoto Co., Inc. (jan. 2013). http://www.ajinomoto.com/en/ir/pdf/Q3-FY12_data_E. pdf, acessada em Junho de 2013.

66. Kinushita, S.; L-Glutamic Acid Production. John Wiley: New York, 2010.

67. Bona, R.; Moser, A.; Acta Biotechnol. 1997, 17, 327.

68. Leuchtenberger, W.; Huthmacher, K.; Drauz, K.; Appl. Microbiol. Biotechnol. 2005, 69, 1 .

69. Chheda, J. N.; Román-Leshkova, Y.; Dumesic, J. A.; Green Chem. 2007, 9, 342 .

70. Sherman, I. L.; Lincoln, I. L.; US Pat. 20130123520, 2013.

71. Schön, M.; Schnürch, M.; Mihovilovic, M. D.; Mol. Divers. 2011, 15, 639.

73. van Putten, R.-J.; van der Waal, J. C.; de Jong, E.; Rasrendra, C. B.; Heeres, H. J.; de Vries, J. G.; Chem. Rev. 2013, 113, 1499.

74. Bredihhin, A.; Mäeorg, U.; Vares, L.; Carbohydr. Res. 2013, 375, 63.

75. James, O. O.; Maity, S.; Usman, L. A.; Ajanaku, K. O.; Ajani, O. O.; Siyanbola, T. O.; Sahu, S.; Chaubey, R.; Energy Environ. Sci. 2010, 3 , 1833. 